\title{
GRAPHENE-BASED SYSTEMS FOR ENHANCED ENERGY STORAGE
}

\author{
Aris Amplianitis ${ }^{(1),(3)}$, Athanasios Masouras ${ }^{(3)}$, Giorgos Gkikas ${ }^{(2)}$, Zampia Kalogridi ${ }^{(1),(2)}$, Athanasios \\ Baltopoulos ${ }^{(2)}$, Antonios Vavouliotis ${ }^{(1),(3)}$, Stavros Tsantzalis ${ }^{(3)}$, Vassilis Kostopoulos ${ }^{(3)}$ and Ugo Lafont ${ }^{(4)}$ \\ (I) PLEIONE ENERGY S.A., www.pleione-energy.com (Greece),Email: amplianitis@pleione-energy.com \\ (2) ADAMANT COMPOSITES Ltd.,www.adamant-composites.gr (GREECE),Email: gkikas@adamant-composites.gr \\ (3) APPLIED MECHANICS \& VIBRATIONS LABORATORY, Dept. Mechanical and Aeronautics Engineering, \\ Polytechnical School, University of Patras, www.aml.mech.upatras.gr (GREECE), Email: \\ kostopoulos@mech.upatras.gr \\ (4) EUROPEAN SPACE AGENCY (ESA), www.esa.int (THE NETHERLANDS),Email: ugo.lafont@esa.int
}

\begin{abstract}
Extensive global research efforts have focused on the exploitation of graphene for enhanced energy storage. Novel graphene-based composite material electrodes have been developed, in many cases with reports of outstanding performance. However, the development of these composites involve extremely complex and costly procedures/methods whose scalability and eventual commercial exploitation is extremely hard [1]. Within the present activity the use of graphene nanotechnology is exploited to manufacture electrodes for supercapacitors. The goal however is to achieve electrodes with increased specific energy density (compared to the currently commercially available products) using proven and simple manufacturing procedures that can easily be scaled-up and offer competitive products. The roadmap was developed under the framework of European Space Agency highlighting the main advantages brought up from this technology. The activity is separated in three parallel routes; the development and test planning of smallscale production of graphene based materials via the tape casting technology, the establishment of a reliable and low cost industrial production process (scale-up) for these materials and the development and testing of an energy storage demonstrator that shall incorporate the novel electrodes and exhibit their favorable characteristics in energy storage applications for use in space.
\end{abstract}

\section{INTRODUCTION}

Graphene is an atomically thin, planar membrane of carbon atoms tightly packed into a two-dimensional (2D) honeycomb lattice with exceptional properties, particularly electronic and electrochemical ones.

As a kind of carbon material, graphene sheet (GS) has attracted increasing attention in a variety of fields because of its high theoretical specific surface area (2600 $\left.\mathrm{m}^{2} \mathrm{~g}^{-1}\right)$, good flexibility, superior chemical/thermal stability, and extraordinary electrical, thermal, and mechanical properties. Its unique structure and associated outstanding properties make graphene highly promising for a wide range of applications in electronics, sensors, and energy storage/conversion. In the case of supercapacitors, the desire for using carbon materials is based on the mechanism of doublelayer capacitance. They store the charges electrostatically using reversible adsorption of ions of the electrolyte onto active materials that are electrochemically stable and have a high accessible surface area. Although graphene has a large theoretical surface area, this area will not result in high electrode capacitance if it is not accessible to ions. Therefore, it should be noted that the capacitance is largely dependent on the layering and stacking configuration of graphene, and much research has been conducted to create graphene structures that display large ionaccessible surface areas [1]. The agglomeration and restacking of graphene nano-sheets during the drying process decrease the electrolyte-ion-accessible surface area, and thus limits the electrochemical performance. To take full advantage of graphene in supercapacitors, dispersing carbon materials, metal oxides/hydroxides, and conducting polymer onto graphene to form graphene-based composites is considered an effective and practical method. These materials will not only prevent the agglomeration and restacking of graphene during the drying process but also contribute to the specific capacitance themselves thus improving the whole supercapacitor performance.

More specifically, transition metal oxides such as $\mathrm{MnO}_{2}$ and $\mathrm{RuO}_{2}$ can exhibit high specific capacitance (pseudocapacitance) via the redox reaction at the interface of the electrode material and the electrolyte [2], [3]. Conducting polymers can offer capacitive behaviors because of the presence of a delocalized $\pi$ electron conjugation system, which can enable fast and reversible redox reactions [4][5]. In addition, conducting polymers also have the advantages of cost effectiveness, environmental benignity, high conductivity in a doped state, and adjustable redox activity through chemical modifications [6]. Thus, conducting polymers are considered as good electrode materials for supercapacitors. However, the limitation of using conducting polymers as standalone materials for supercapacitors is that they can rapidly degrade as a 
result of swelling and shrinkage, resulting in poor cycle stability [1]. The introduction of conducting polymers into graphene not only can prevent the agglomeration and restacking of graphene during the drying process to enhance the EDL capacitance of graphene but also can improve the mechanical and electrochemical cycling stability of the polymers. Thus, a combination of graphene and electrically conducting polymers is beneficial for improving the electrochemical performance. A variety of polymers such as polypyrrole (PPy), polyaniline (PANI), poly-3,4ethylenedioxythiophene (PEDOT) and polythiphene (PT) grown on the graphene sheets have been investigated for electrode materials [5].

In the present work three types of graphene based composite materials are developed and tested for incorporation in supercapacitors: Graphene Nanoplatelets (GNPs) with PVDF binder composite, GNPs with PVDF and transition metal oxide $\mathrm{MnO}_{2}$ and GNPs with PVDF and conductive polymer PANI

On a later stage of the activity further investigation will be performed for the favorable characteristics of these materials and how could efficiently be scaled-up to produce large films for industrial incorporation in energy storage devices.

\section{MATERIALS DEVELOPMENT AND TESTING}

Since the discovery of graphene, many routes for its synthesis have been reported. Two main routes are widely available: either exfoliation of natural or synthetic graphite using, mechanical, chemical, liquidphase or electrochemical exfoliation techniques, or direct formation methods from organic molecules such as methane and other hydrocarbon sources [1]. While the second method can produce high-quality graphene films, it is not suitable for large scale economical production of graphene mainly due to it high cost [7]. Chemical exfoliation, on the other hand, has the advantages of scalability and low cost although it is difficult to obtain single-layer high-quality graphene because of the defect creation during exfoliation [8]. In the present work, low cost chemically reduced Graphene Nanoplatelets (GNPs) where employed with an overall thickness of approximately 3-10 nanometers (approx. 4 layers thick). As the main goal of the activity is to employ cheap and scalable production of composite films, the tape casting method was chosen. Slurry preparation starts with magnetic stirring of the polymeric binder (PVDF) with NMP solvent, followed by nanoparticle dispersion and thorough sonication of the mixture. From then on, the process involves the feeding of slurry in front of a doctor blade and the formation of a film with the required thickness. A drying stage follows where the solvent evaporates.
While the followed procedure for tape casting electrodes from a slurry is not innovative, additional production steps were applied during the development to improve the structural integrity of the films. Annealing of the materials after evaporation of the solvent was performed at a temperature closer to the melting point of PVDF, at this stage the crystallization of PVDF produces an electroactive phase of crystallinity ( $\beta$-phase) which is necessary for energy applications [9].

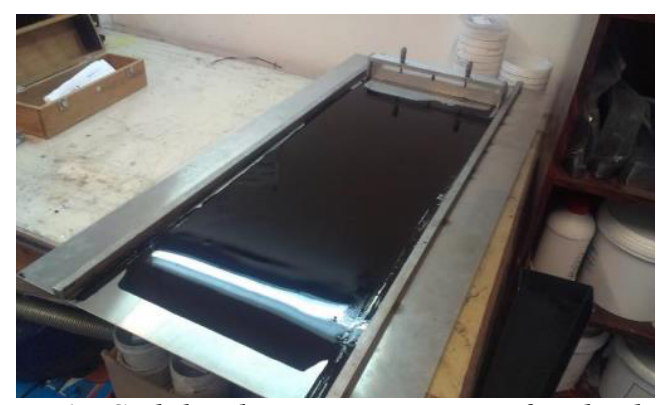

Figure 1. Stabilized casting process for buckyfilms production

The developed composites and their respective compositions are:

- GNP/PVDF: $80 \mathrm{wt} \%$ Graphene Nano-platelets (GNPs) with $20 \mathrm{wt} \%$ PVDF Binder

- GNP/PANI: 76 wt $\%$ GNPs, 19 wt $\%$ PVDF and $5 \mathrm{wt} \% \mathrm{PANI}$

- GNP/MnO $2: 73 \mathrm{wt} \%$ GNPs, $18 \mathrm{wt} \% \mathrm{PVDF}$ and $9 \mathrm{wt} \% \mathrm{MnO}_{2}$

The properties evaluation of the produced materials includes physical characterization, electrical resistivity measurements and electrochemical characterization which is necessary for materials intended for energy storage applications.

Morphology texture of the produced composite was investigated by Scanning Electron Microscopy (SEM, LEO SUPRA 35VP).

The surface and through the thickness electrical resistance of the produced materials was investigated via four probe measurement methods (surface and through thickness).

Electrochemical performance (electrode capacitance) of the materials was investigated by Cyclic Voltammetry in a 3-electrode configuration set-up using an AUTOLAB potentiostat/galvanostat analyzer. Tests were performed on a sample with active area of $0.7 \mathrm{~cm}^{2}$ and active material mass of $15 \mathrm{mg}$. The electrolyte was $\mathrm{KOH}$ solutions of 0,5 and $2 \mathrm{M}$ and the scan rates were adjusted at 10, 20, 30, 40, 50, 100, $200 \mathrm{mV} / \mathrm{s} . \mathrm{Ag} / \mathrm{AgCl}$ reference electrode was used. The specific capacitance 
of the electrodes was calculated using Eq.1.

$$
C=\int_{E_{1}}^{E_{2}} i(E) d E / 2\left(E_{2}-E_{1}\right) m v
$$

where $\int \mathrm{i}(\mathrm{E}) \mathrm{dE}$ is the total voltammetric charge obtained by integration of positive and negative sweep in cyclic voltammetry graphs, (E2 - E1) is the potential window width, $\mathrm{m}$ is the mass, $\mathrm{v}$ is the scan rate.

In the first phase of the activity, various film production parameters were tested in order to optimize both the procedure and the quality of the produced films. Optimum active material contents were also investigated. In later stage (results not presented here) the finalized electrodes shall be incorporated in actual supercapacitors cells (together with appropriate electrolyte) and Galvanostatic charge and discharge cyclic experiments are scheduled to be performed.

For the aforementioned purposes, an appropriate laboratory cell enclosure has been manufactured, with the ability to accommodate both 2 and 3 -electrode setups, as shown in Figure 2.

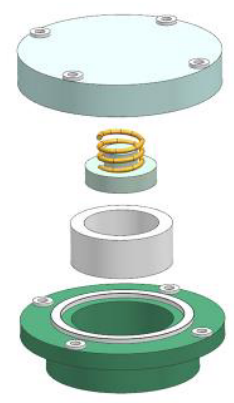

(a)

Figure 2. (a) Blueprint of the cell configuration and (b)Two-electrode cell

\section{RESULTS AND DISCUSION}

3.1. Self - Standing attributes of the produced materials

One of the targets of this activity is the production of self-standing electrodes for energy storage applications. The advanced mechanical properties of GNPs and their high surface area are promising characteristics to achieve the production of self-standing GNP films with structural integrity and energy storage performance (specific capacitance and capacity). The first results proved that it is feasible to produce self-standing electrodes with $80 \%$ wt. content of GNPs (Figure 3):

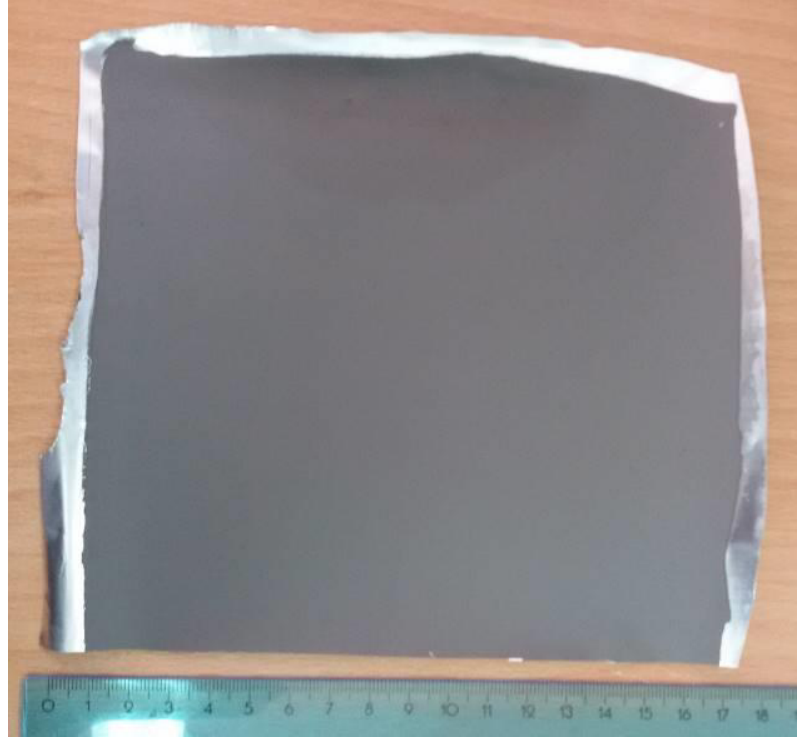

(a)

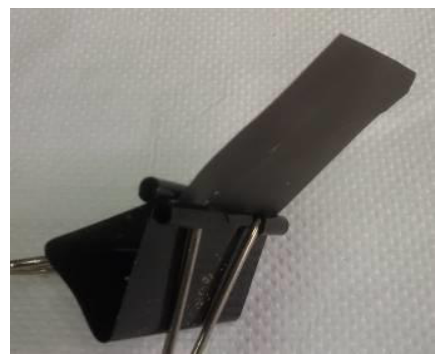

(b)

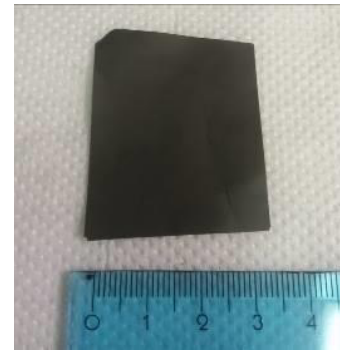

(c)

Figure 3. (a) GNP film after manufacturing on aluminum substrate. (b), (c) Self-standing GNP-film (electrode)

\subsection{Microstructural Analysis}

Microstructural analysis of the produced materials was performed by SEM. Several micrographs captured in different magnifications in order to investigate the internal structure of the produced films.

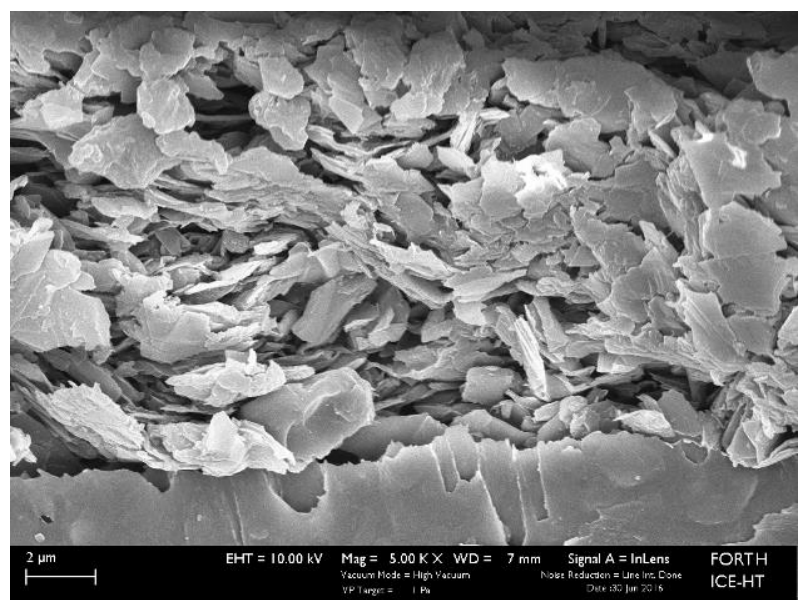

Figure 4. Magnification $5 K x-$ Closer view of the internal microstructure of GNP/PVDF composite 


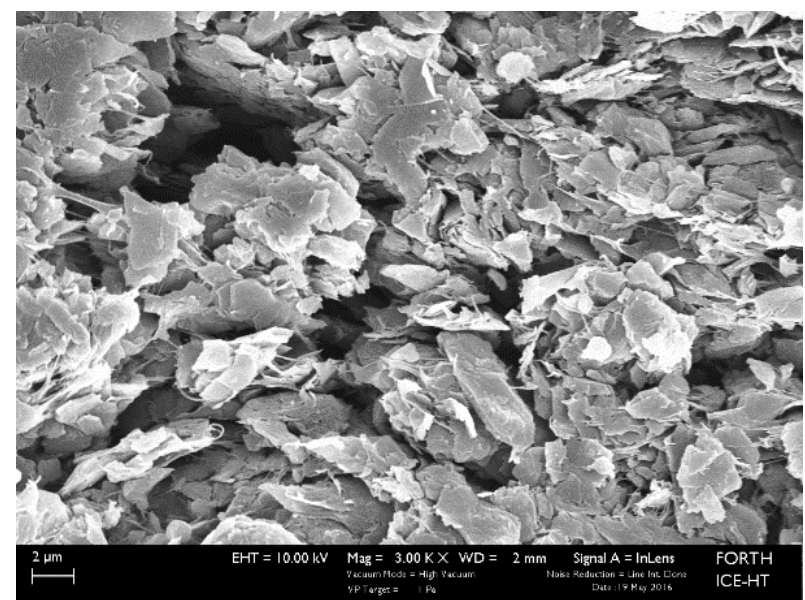

Figure 5. Magnification $3 K x-$ Close view of the internal microstructure of the GNP/PANI composite.

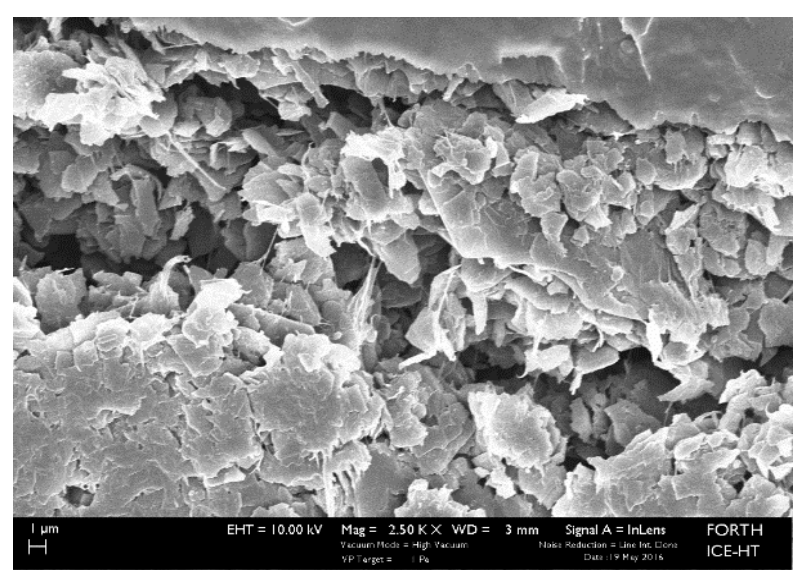

Figure 6. Magnification 2.5Kx - Closer view of the internal microstructure of $\mathrm{GNP} / \mathrm{MnO}_{2}$ composite.

In general, the microstructure of the films that were produced is uniform and the GNPs are well distributed in the structure exhibiting an inter-particular porosity. This intrinsic porosity is beneficial in that it will allow better electrode/electrolyte contact.

\subsection{Electrical Resistivity Results}

Electrical resistance of the samples was measured for the evaluation of their electrical behaviour. Electrical resistance of electrodes must be low to avoid high internal resistance of the future supercapacitor cell. Figure 7. Through the thickness electrical resistance of the produced electrodes $(\Omega)$.Figure 7 presents the results of electrical resistance through the thickness of the produced composites.

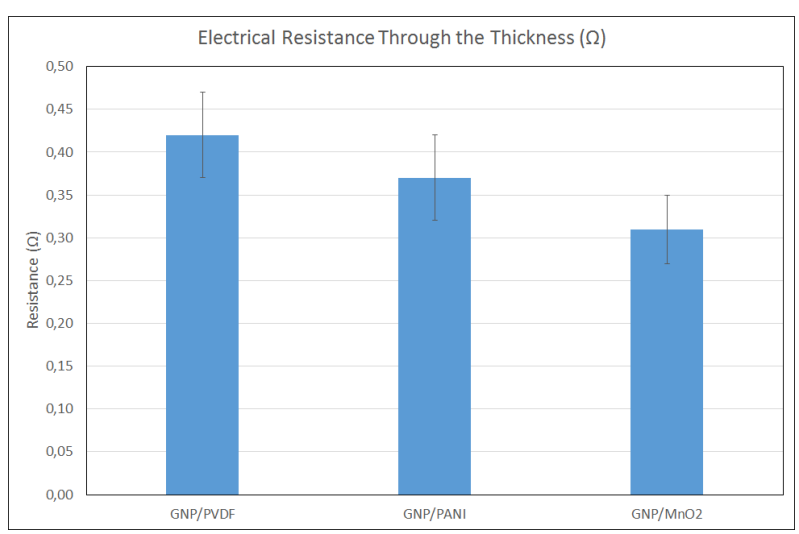

Figure 7. Through the thickness electrical resistance of the produced electrodes $(\Omega)$.

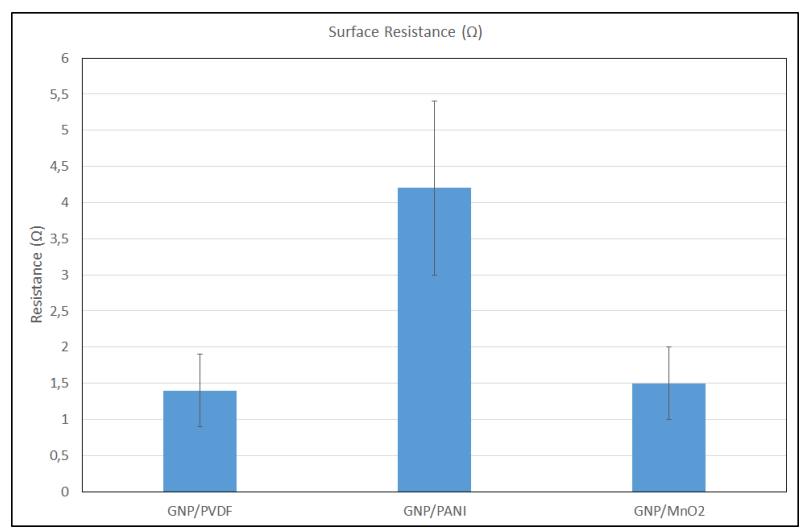

Figure 8. Surface electrical resistance of produced electrodes $(\Omega)$.

\subsection{Cyclic Voltammetry Results}

During this test campaign, it was achieved to extract one set of clear measurements for the three different materials developed.

The cyclic voltammetry tests on the GNP/PVDF composite were performed on samples with active area of $0.7 \mathrm{~cm}^{2}$ and active material mass $15 \mathrm{mg}$. The electrolyte was $2 \mathrm{M} \mathrm{KOH}$ solution and the scan rates was adjusted at 10, 20, 30, 40, 50, 100, $200 \mathrm{mV} / \mathrm{s}$. $\mathrm{Ag} / \mathrm{AgCl}$ reference electrode was used. The results are presented in Figure 9 and Figure 10. 


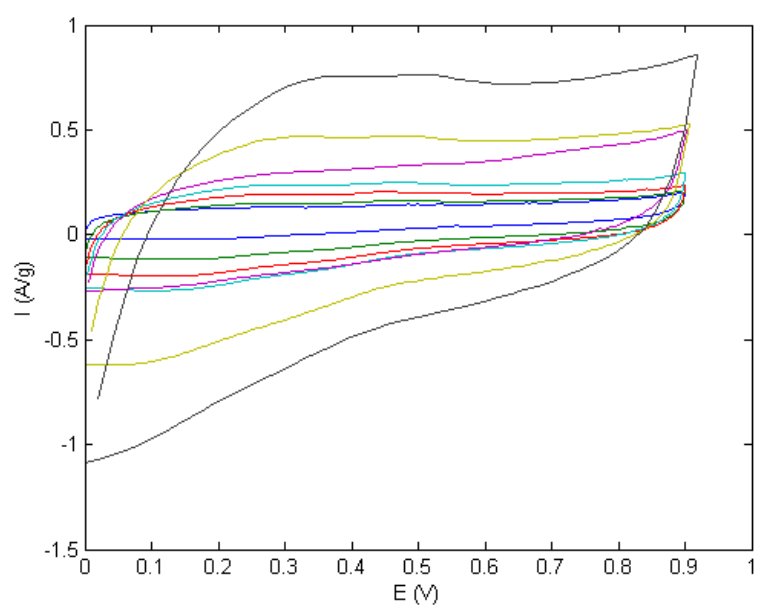

Figure 9. Working Electrode Current vs Reference Electrode Potential for the GNP/PVDF composite.

Scan Rates: 10(—), 20(—), 30(—), 40(—), 50(—), $100(-), 200(-) \mathrm{mV} / \mathrm{s}$

In Figure 9. it is observed that up until the scan rate of $100 \mathrm{mV} / \mathrm{s}$, the CVs retain a nearly symmetrical rectangular shape within the range of scan rates employed, which is characteristic of electro-chemical double-layer capacitive behaviour [10]. Furthermore, no great peaks appear which indicates that the supercapacitive behaviour is free from redox reactions or is purely based on the electrostatic mechanism. On the contrary the GNP/PANI and $\mathrm{GNP} / \mathrm{MnO}_{2}$ electrodes exhibit increasing peaks with increased scan rate and departure for the symmetrical rectangular shape due to the presence of pseudo-capacitive activity of the PANI and metal oxide.

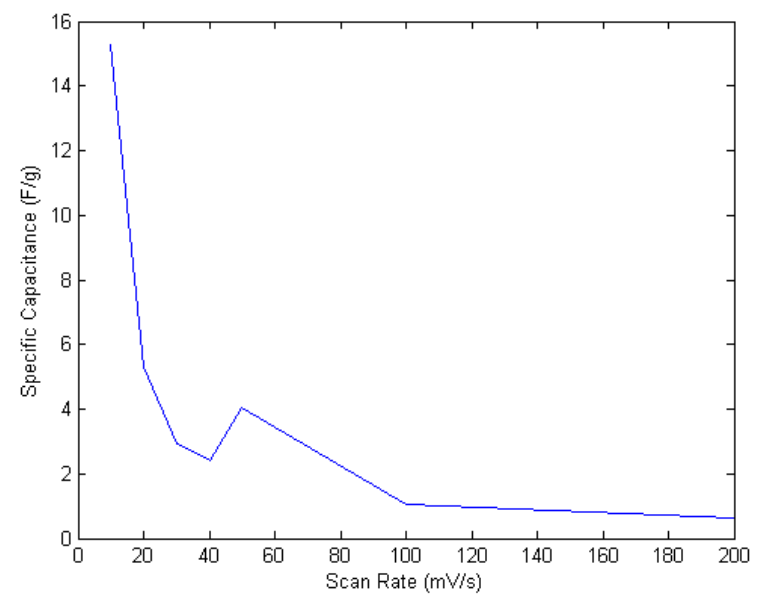

Figure 10. Evolution of the specific capacitance as function of the scan rate for the GNP/PVDF composite electrode
Results of the cyclic voltammetry tests performed on the GNP/PANI composite are presented in Figure 11 and Figure 12.

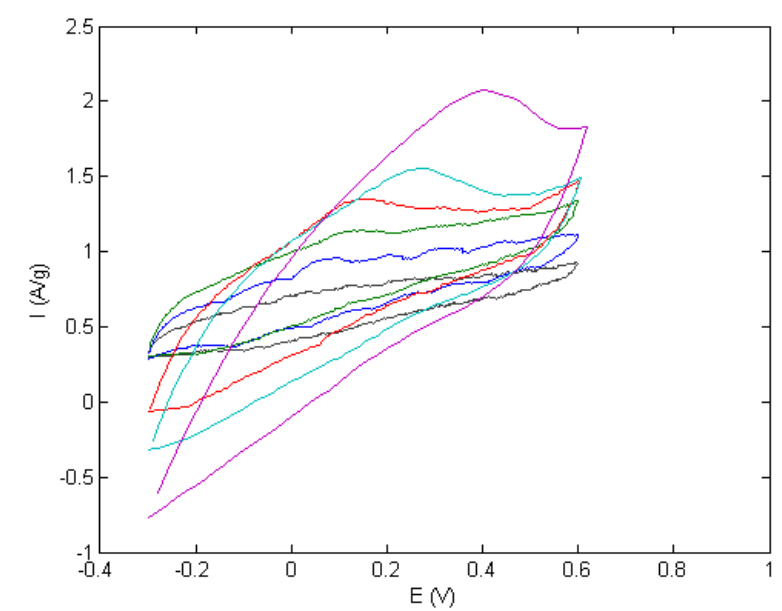

Figure 11. Working Electrode Current vs Reference Electrode Potential for the GNP/PANI composite.

Scan Rates: 10( $($ ), 20( ), 30( $200(-) \mathrm{mV} / \mathrm{s}$

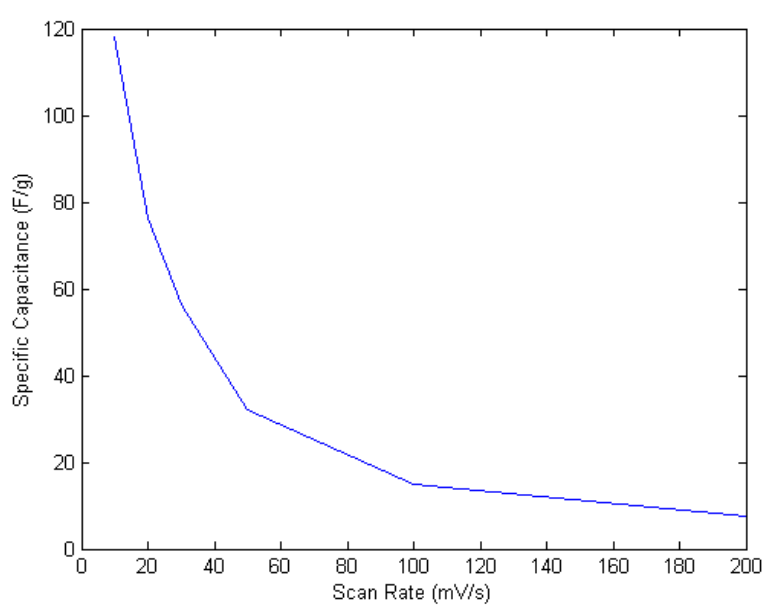

Figure 12. Evolution of the specific capacitance as function of the scan rate for the GNP/PANI composite electrode.

Finally, similar cyclic voltammetry tests were also performed on the GNP/ $\mathrm{MnO}_{2}$ composite. The results are presented in Figure 13 and Figure 14. 


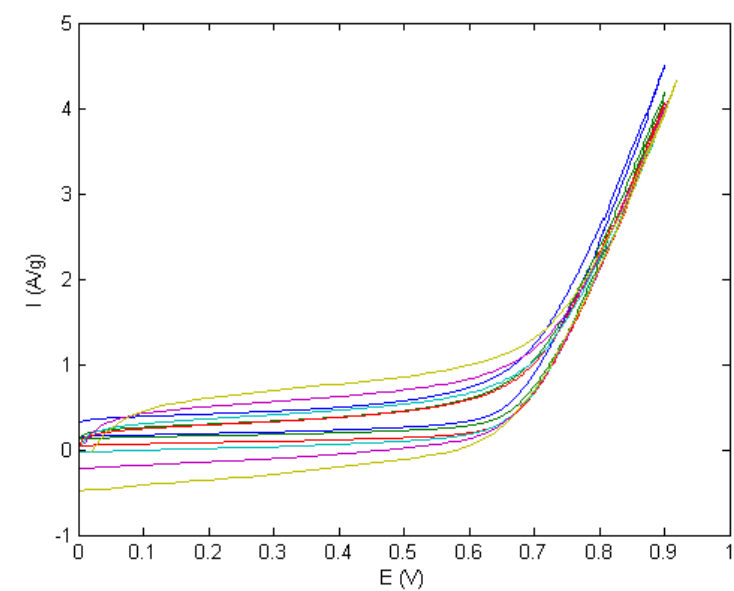

Figure 13. Working Electrode Current vs Reference Electrode Potential for the GNP/MnO $\mathrm{O}_{2}$ composite. Scan Rates: 10(—), 20(-), 30(-), 50( ), 100( ), $200(-) \mathrm{mV} / \mathrm{s}$

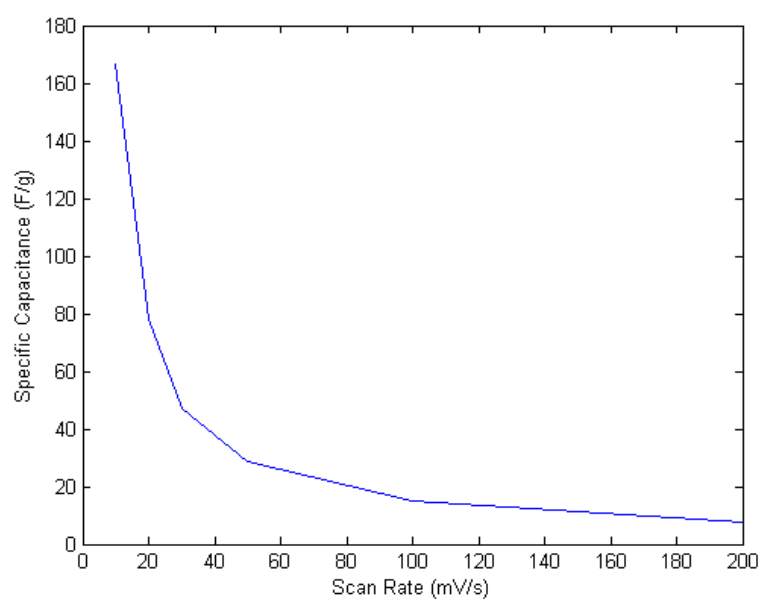

Figure 14. Evolution of the specific capacitance as function of the scan rate for $\mathrm{GNP} / \mathrm{MnO}_{2}$ composite electrode

Figures 10,12 and 14 present the exhibited decrease of specific capacitance with increasing CV scan rate. This decrease has to do with reaction kinetics between the electrode and the electrolyte and therefore becomes more apparent in the electrodes that involve slower faradic reactions such as the $\mathrm{GNP} / \mathrm{MnO}_{2}$. These faradic reactions however are the reason these electrodes exhibit high pseudocapacitance.

Comparison of the specific capacitance results are presented below (at scan rate: $10 \mathrm{mv} / \mathrm{s}$ ) in Figure 15 . Incorporation of PANI and $\mathrm{MnO}_{2}$ improve the specific capacitance of the electrode from the base value of the reference electrode only with GNPs. Specific capacitance enhanced over an order of magnitude by the presence of PANI and $\mathrm{MnO}_{2}$ but still further improvement is needed.

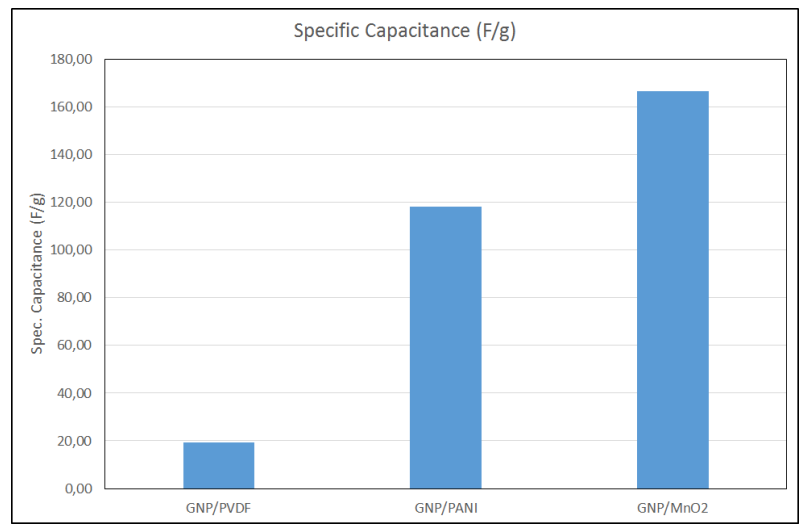

Figure 15. Specific Capacitance results at scan rate of $10 \mathrm{mv} / \mathrm{s}$

\section{PROCESS INDUSTRIALIZATION}

Next essential part of the project is the investigation and development of a scale-up manufacturing procedure for the selected graphene based composite materials. Scaling up the production while at the same time maintaining the favorable performance of these materials is the ultimate goal towards a commercialization plan that involves the incorporation of these materials in high energy density supercapacitors. To achieve that, parameters such as auto-feeding of the slurry on the doctor blade, velocity of the blade for the filming, on site drying without removing the final films from the casting substrate were investigated and a small semi-automated production line was designed and manufactured. Currently the automated procedure is being fine-tuned and the first electrodes sheets that are being produced are being tested for their performance. A blueprint of the final film applicator is shown in Figure 16.

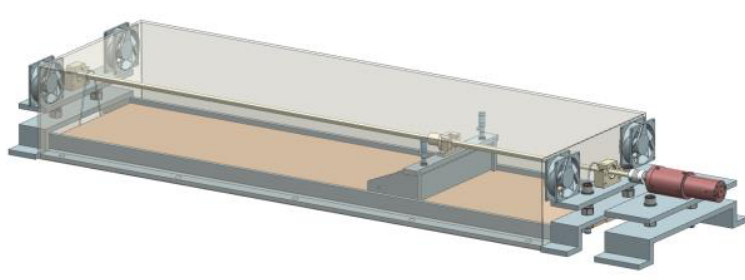

Figure 16. Blueprint of the film applicator device

\section{CONCLUSION}

Within the present activity the production of graphene based composite electrodes using a proven and economical tape casting method is investigated in order 
to later establish a scale-up manufacturing procedure for supercapacitor electrodes. Initial 3-electrode CV measurements of the laboratory scale samples exhibit the favorable characteristics of the graphene based electrodes with a maximum specific capacitance of 165 $\mathrm{Fg}^{-1}$ for the Graphene/ $\mathrm{MnO}_{2}$ composite. Furthermore, the produced electrodes are free-standing and flexible. In relevant works, specific capacitances of Graphene $/ \mathrm{MnO}_{2}$ composite electrodes are reported at $130 \mathrm{Fg}^{-1}$ [10], $180 \mathrm{Fg}^{-1}$ [2] and $216 \mathrm{Fg}^{-1}$ [11]. However the capacitance of a supercapacitor is strongly dependent on the cell configuration used for the electrochemical measurement and it is always significantly higher when using a three-electrode system[12]. Therefore, in order to have a clearer image of their performance, the produced electrodes need to be integrated in 2-electrode cells and measured during Galvanostatic charging and discharging.

Efforts will now focus on further improvements to achieve higher specific capacitance for the produced materials while keeping the production cost low. First steps towards the establishment of a reliable and low cost industrial production process (scale-up) for these materials has been achieved and the first electrodes sheets that are being produced will need to be tested for their performance before the scale up can be considered successful. These results will be reported in future work when the entire project finalizes and concludes.

\section{AKNOWLEDGMENTS}

- Cyclic voltammetry testing of the produced composites was done at the Laboratory of Chemical \& Electrochemical Processes of the Department of Chemical Engineering, University of Patras.

- The work reported in the present document pertains to the ESA commissioned activity "Graphene-based systems for enhanced energy storage" (ESA-GADGET-4000112857/14/NL /PA-CCN1).

\section{REFERENCES}

[1] A. Rashid bin Mohd Yusoff, Graphene-Based Energy Devices. 2015.

[2] J. Yan, Z. Fan, T. Wei, W. Qian, M. Zhang, and F. Wei, "Fast and reversible surface redox reaction of graphene- $\mathrm{MnO} 2$ composites as supercapacitor electrodes," Carbon N. Y., vol. 48, no. 13, pp. 3825-3833, Nov. 2010.

[3] G. Yu, L. Hu, N. Liu, H. Wang, M. Vosgueritchian, Y. Yang, Y. Cui, and Z. Bao, "Enhancing the supercapacitor performance of graphene/MnO2 nanostructured electrodes by conductive wrapping," Nano Lett., vol. 11, no. 10, pp. 4438-4442, 2011.
[4] M. Liang and L. Zhi, "Graphene-based electrode materials for rechargeable lithium batteries," J. Mater. Chem., vol. 19, no. 33, p. 5871, 2009.

[5] H. Gómez, M. K. Ram, F. Alvi, P. Villalba, E. Stefanakos, and A. Kumar, "Grapheneconducting polymer nanocomposite as novel electrode for supercapacitors," J. Power Sources, vol. 196, no. 8, pp. 4102-4108, 2011.

[6] Z. Song, T. Xu, M. L. Gordin, Y. B. Jiang, I. T. Bae, Q. Xiao, H. Zhan, J. Liu, and D. Wang, "Polymer-graphene nanocomposites as ultrafastcharge and -discharge cathodes for rechargeable lithium batteries," Nano Lett., vol. 12, no. 5, pp. 2205-2211, 2012.

[7] L. Zhaoping and Z. Xufeng, Graphene, Energy storage and conversion Application. CRC Press Taylor \& Francis Group.

[8] M. D. Stoller, S. Park, Y. Zhu, J. An, and R. S. Ruoff, "Graphene-Based Ultracapacitors," pp. 6-10, 2008.

[9] P. Martins, A. C. Lopes, and S. LancerosMendez, "Electroactive phases of poly(vinylidene fluoride): Determination, processing and applications," Prog. Polym. Sci., vol. 39, no. 4, pp. 683-706, 2014.

[10] Y. He, W. Chen, X. Li, Z. Zhang, J. Fu, C. Zhao, and E. Xie, "Freestanding threedimensional graphene/Mno2 composite networks as ultralight and flexible supercapacitor electrodes," ACS Nano, vol. 7, no. 1, pp. 174-182, 2013.

[11] S. Chen, J. Zhu, X. Wu, Q. Han, and X. Wang, "Graphene oxide-Mno2 nanocomposites for supercapacitors," ACS Nano, vol. 4, no. 5, pp. 2822-2830, 2010.

[12] Q. Cheng, J. Tang, J. Ma, H. Zhang, N. Shinya, and L.-C. Qin, "Graphene and carbon nanotube composite electrodes for supercapacitors with ultra-high energy density.," Phys. Chem. Chem. Phys., vol. 13, no. 39, pp. 17615-24, 2011. 